\title{
sciendo
}

ISSN: $1231-4005$

e-ISSN: $2354-0133$

DOI: $10.2478 /$ kones-2019-0105

\section{OPERATING PARAMETERS OF A SLIDE BEARING WITH PARABOLIC-SHAPED SLIDE SURFACES WITH CONSIDERATION OF THE STOCHASTIC CHANGES IN THE LUBRICATION GAP HEIGHT}

\author{
Andrzej Miszczak \\ Gdynia Maritime University \\ Faculty of Marine Engineering \\ Morska Street 81-87, 81-225 Gdynia, Poland \\ tel.: +4858 5586348, fax: +48585586399 \\ e-mail:a.miszczak@wm.umg.edu.pl \\ Krzysztof Wierzcholski \\ Technical University of Koszalin \\ Faculty of Technology and Education \\ Śniadeckich Street 2, 75-453 Koszalin, Poland \\ tel.: +48943478344, fax: +48943426753 \\ e-mail: krzysztof.wierzcholski@wp.pl
}

\begin{abstract}
In this article, the authors present the equations of the hydrodynamic theory for a slide bearing with parabolicshaped slide surfaces. The lubricating oil is characterized by non-Newtonian properties, i.e. an oil for which, apart from the classic oil viscosity dependence on pressure and temperature, also an effect of the shear rate is taken into account. The first order constitutive equation was adopted for considerations, where the apparent viscosity was described by the Cross equation. The analytical solution uses stochastic equations of the momentum conservation law, the stream continuity and the energy conservation law. The solution takes into account the expected values of the hydrodynamic pressure $E X[p(\varphi, \zeta)]$, of the temperature $E X[T(\varphi, y, \zeta)]$, of the velocity value of lubricating oil

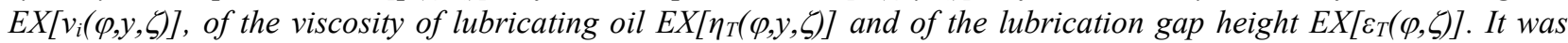
assumed, that the oil is incompressible and the changes in its density and thermal conductivity were omitted. A flow of lubricating oil was laminar and non-isothermal. The research concerned the parabolic slide bearing of finite length, with a smooth sleeve surface, with a full wrap angle. The aim of this work is to derive the stochastic equations, that allow to determine the temperature distribution, hydrodynamic pressure distribution, velocity vector components, load carrying capacity, friction force and friction coefficient, in the parabolic sliding bearing, lubricated with nonNewton (Cross) oil, including the stochastic changes in the lubrication gap height. The paper presents the results of analytical and numerical calculation of flow and operating parameters in parabolic sliding bearings, taking into account the stochastic height of the lubrication gap. Numerical calculations were performed using the method of successive approximations and finite differences, with own calculation procedures and the Mathcad 15 software.
\end{abstract}

Keywords: hydrodynamic lubrication, analytical stochastic principles and solutions, apparent viscosity, numerical calculation, load carrying capacity, friction force

\section{Introduction}

The operating parameters of a slide bearing depend on many factors and mainly on hydrodynamic pressure, temperature, dynamic viscosity of the lubricant $[1-3,5-7,9,10]$. It is also important, whether the oil has Newtonian or non-Newtonian properties $[1-3,5,6,9,10]$. These values are influenced, among others, by the type of slide bearing and operating conditions (vibrations, turbulence, stationary or non-stationary lubrication, etc.). Some of these factors change 
randomly (vibrations, surface roughness, loads). Some previous research efforts have been limited to consideration of the impact of stochastic changes in the bearing gap height on the pressure distribution $[5,6,9]$. This fact has become the aspiration of the authors to conduct tribological tests in a comprehensive approach, and consider stochastic changes in lubrication gap height, velocity and viscosity of lubricating oil, oil temperature, bearing load capacity, friction forces and coefficient of friction.

Investigations of stochastic processes consist in selecting the most probable value of the considered parameter. For this purpose, the expected values of the gap height and viscosity function are determined. The expected value is then the most probable value of the height of the bearing gap or viscosity. This value is necessary to determine the lubrication parameters, in particular the hydrodynamic pressure, friction forces and coefficient of friction. The probabilistic description of the friction and lubrication process also requires the determination of standard deviations for the above-mentioned necessary conditions.

The authors investigated the parabolic bearing with the full wrap angle. The flow of the lubricant in the gap is stationary, laminar and non-isothermal.

The aim of this research is to investigate the impact of stochastic changes in lubrication gap height on the oil viscosity values and flow velocity, and on the hydrodynamic pressure, friction forces and friction coefficient.

\section{Stochastic height of the bearing lubrication gap}

A parabolic bearing gap, limited from one side to the surface of the bearing shaft, and the other to the surface of the bearing sleeve, is filled with lubricating oil with non-Newtonian properties. We assume a characteristic constant dimensional value of the height of the lubrication gap $\varepsilon_{o}$ and dimensionless gap height function $\varepsilon_{1}$ dependent on $\varphi$ and on $\zeta_{1}$. This function is the sum of the two parts described by the formula (1) [10]:

$$
\varepsilon_{1}=\varepsilon_{T} / \varepsilon_{o}=\varepsilon_{1 p}\left(\varphi, \zeta_{1}\right)\left[1+\delta_{1}(\varphi, \zeta, \xi)\right] .
$$

The symbol $\varepsilon_{1 p}$ is the dimensionless gap height limited by nominally smooth bearing surfaces. The symbol $\xi$ describes the random variable, which characterizes the roughness arrangement. The random changes in the gap height $\varepsilon_{T}$ are caused by the stochastic distribution of peaks and valleys at bearing shaft and sleeve surfaces. The parameter $\delta_{1}$ is the dimensionless stochastic variable of corrections for the gap height. The corrections of random changes refer to the height of the gap $\varepsilon_{T}$, but the concern the apparent viscosity $\eta_{a}$, hydrodynamic pressure, temperature and other parameters.

The geometry of the lubrication gap is shown in Fig. 1, when, the function describing its height without taking into account the misalignment, can be written in the following form:

$$
\varepsilon_{p}=\varepsilon_{o} \varepsilon_{1 p}=\varepsilon_{o}(1+\lambda \cos \varphi) \text {. }
$$

\section{Basic equations in parabolic coordinates}

The equation of momentum conservation, the equation of stream continuity and the equation of energy conservation will be written in a parabolic coordinate system $\varphi, y, \zeta$. We will take into account the expected values of hydrodynamic pressure $E X[p(\varphi, \zeta)]$, temperature $E X[T(\varphi, y, \zeta)]$, lubricating oil velocity value $E X\left[v_{i}(\varphi, y, \zeta)\right]$, lubricating oil viscosity $E X\left[\eta_{a}(\varphi, y, \zeta)\right]$, lubrication gap height $E X\left[\varepsilon_{T}(\varphi, \zeta)\right]$. We assume that the lubricating oil is incompressible and we omit changes in lubricating oil density. Then, we use the classic simplification of hydrodynamic equations for the boundary layer, consisting in omitting the part of equations, which are of the order of a radial relative clearance, i.e. $10^{-4}-10^{-3}$. 

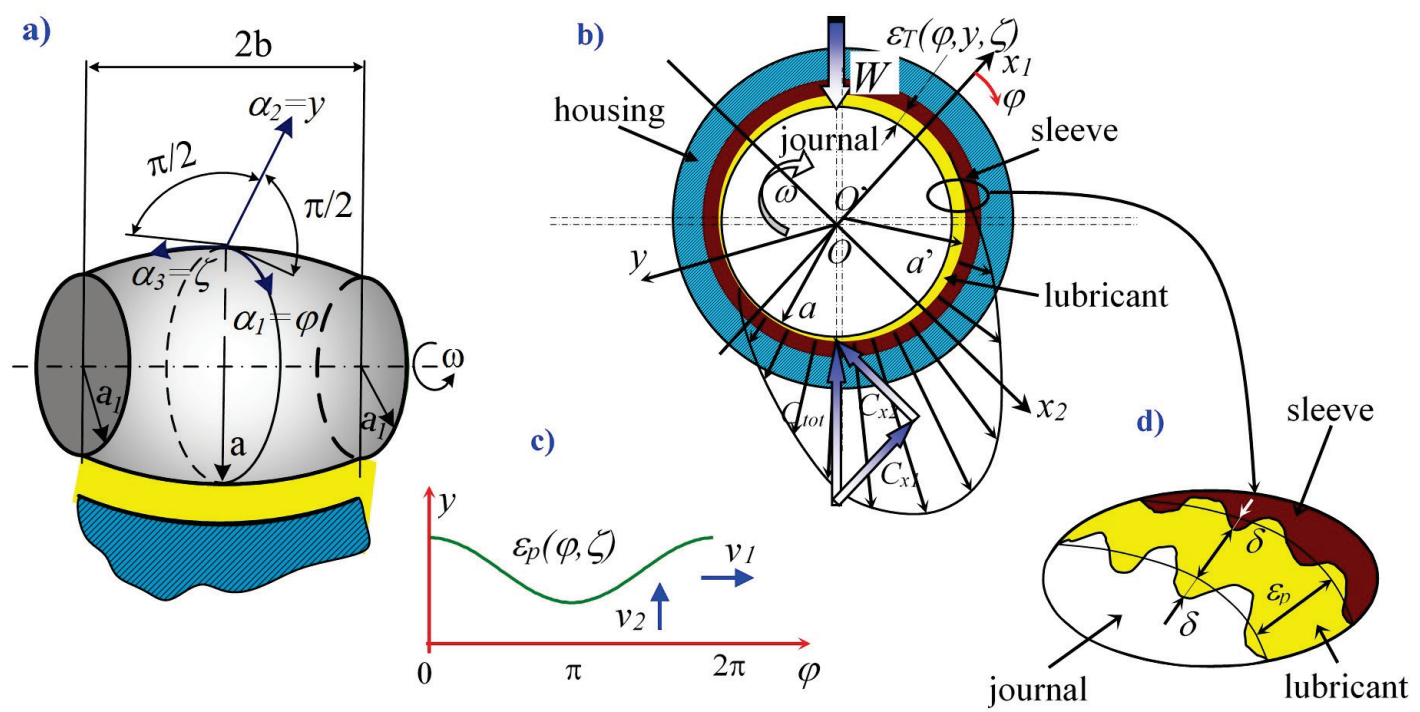

Fig. 1. The geometry of parabolic slide bearing

The system of partial differential equations determines the following expected values of randomly variable unknown functions: three components of the lubricating oil velocity vector $E X\left[v_{\mathrm{i}}(\varphi, y, \zeta)\right][\mathrm{m} / \mathrm{s}]$; hydrodynamic pressure $E X[p(\varphi, \zeta)][\mathrm{Pa}]$, temperature $E X[T(\varphi, y, \zeta)][\mathrm{K}]$.

The part on the equation of momentum conservation describes the centrifugal forces occurring during the cooperation of two surfaces. These forces occur only when the Lame's coefficient $h_{1}$ is a function of the $\zeta$ coordinate. This is the case for surfaces with a spherical, conical, parabolic or elliptical shape, but not cylindrical, where the coefficient $h_{1}$ has a constant value. By analysing the influence of centrifugal forces on the value of hydrodynamic pressure, it can be stated, that for angular velocities in the range from 0 to $300 \mathrm{~s}^{-1}$ and for the differences in diameters of the parabolic shaft (see Fig. 1) $a / a_{1}$ up to $30 \%$, this effect is of the order of $1-3 \%$. Due to the above, this effect was omitted in further analysis.

The integration of the system of equations (equation of momentum conservation, the equation of stream continuity and the equation of energy conservation), describing the lubrication of the rotating surfaces separated by a thin layer of a lubricating oil, will be carried out in the curvilinear coordinates $(\varphi, y, \zeta)$ of the parabolic coordinate system.

The shaft surface rotates at a constant angular speed $\omega$, while the sleeve surface is fixed. The volume between these surfaces is filled with lubricating oil and the distance between them is determined by the random height $\varepsilon_{T}$ variable. Therefore, the boundary conditions for the expected velocity components functions of the lubricating oil $\operatorname{EX}\left(v_{1}\right), \operatorname{EX}\left(v_{2}\right), \operatorname{EX}\left(v_{3}\right)$ in the $\varphi, y, \zeta$ directions, are as follows:

$$
\begin{gathered}
E X\left(v_{1}\right)=\omega a \cos ^{2}\left(\Lambda_{p 1}, \zeta_{1}\right) \text { for } y=0, E X\left(v_{1}\right)=0 \text { for } y=E X\left(\varepsilon_{T}\right), \\
E X\left(v_{2}\right)=0 \text { for } y=0, \text { and } E X\left(v_{2}\right)=0 \text { for } y=E X\left(\varepsilon_{T}\right), \\
E X\left(v_{3}\right)=0 \text { for } y=0, E X\left(v_{3}\right)=0 \text { for } y=E X\left(\varepsilon_{T}\right) .
\end{gathered}
$$

By applying the condition (3) to the appropriate equations of momentum conservation, we obtain the following form of the expected function of the randomly variable velocity vector component in the circumferential direction $\varphi$ :

$$
E X\left[v_{1}(\varphi, y, \zeta)\right]=\left(\frac{1}{h_{1}(\zeta)} \frac{\partial E X(p)}{\partial \varphi}\right) A_{\eta}+\left(1-A_{s}\right) \omega h_{1}\left(\zeta_{1}\right),
$$

where the functions $A_{s}[1], A_{\eta}\left[\mathrm{m}^{4} / \mathrm{Ns}\right]$ have been introduced, which are as follows: 


$$
A_{s}(\varphi, y, \zeta) \equiv \frac{\int_{0}^{y} \frac{1}{E X\left(\eta_{a}\right)} d y}{\int_{0}^{E X\left(\varepsilon_{T}\right)} \frac{1}{E X\left(\eta_{a}\right)} d y}, \quad A_{\eta}(\varphi, y, \zeta) \equiv \int_{0}^{y} \frac{y}{E X\left(\eta_{a}\right)} d y-\frac{\left(\int_{0}^{y} \frac{1}{E X\left(\eta_{a}\right)} d y\right)\left(\int_{0}^{E X\left(\varepsilon_{T}\right)} \frac{y}{E X\left(\eta_{a}\right)} d y\right)}{\int_{0}^{E X\left(\varepsilon_{T}\right)} \frac{1}{E X\left(\eta_{a}\right)} d y},
$$

for: $0 \leq \varphi \leq 2 \pi,-b \leq \zeta \leq+b, 0 \leq y \leq E X\left(\varepsilon_{T}\right), E X\left(\varepsilon_{T}\right)=E X\left[\varepsilon_{T}(\varphi, \zeta)\right], E X\left(\eta_{a}\right)=E X\left[\eta_{a}(\varphi, y, \zeta)\right]$, where the expected value of apparent viscosity $\eta_{a}[\mathrm{Pas}]$ is in the form:

$$
E X\left[\eta_{a}(\varphi, r, z)\right]=E X\left[\eta_{a}(p, T, \dot{\gamma})\right] \equiv \eta_{o} E X\left(\eta_{1 p} \cdot \eta_{1 T} \cdot \eta_{1 \gamma}\right),
$$

while:

$$
\begin{gathered}
E X\left[\eta_{1 p}(\varphi, r, z)\right]=E X\left(\frac{\eta_{h p}}{\eta_{o}}-\frac{\eta_{o}-\eta_{h p}}{\eta_{o}} e^{-\delta_{p} p}\right), E X\left[\eta_{1 T}(\varphi, r, z)\right]=E X\left(e^{-\delta_{T}\left(T-T_{o}\right)}\right), \\
E X\left[\eta_{1 \gamma}(\varphi, r, z)\right]=E X\left(\frac{\eta_{\infty}}{\eta_{o}}+\frac{1-\frac{\eta_{\infty}}{\eta_{o}}}{1+(d \cdot \dot{\gamma})^{n}}\right), \dot{\gamma} \equiv\left[\frac{1}{2} \cdot \operatorname{tr}\left(A_{1}^{2}\right)\right]^{\frac{1}{2}}, \\
\Lambda_{p 1} \equiv \sqrt{\frac{a-a_{1}}{a}}, \zeta_{1}=\zeta / b, h_{1}=a \cos ^{2}\left(\Lambda_{p 1} \zeta_{1}\right), h_{3}=\sqrt{1+4\left(\Lambda_{p 1} / L_{1}\right)^{2} \sin ^{2}\left(\Lambda_{p 1} \zeta_{1}\right)} \cos \left(\Lambda_{p 1} \zeta_{1}\right) .
\end{gathered}
$$

The following designations are introduced: $2 b-$ the length of bearing sleeve [m], $\rho$ - lubricating oil density $\left[\mathrm{kg} / \mathrm{m}^{3}\right], \eta_{h p}$ - lubricating oil dynamic viscosity at high pressure [Pas], $\eta_{\infty}$ - lubricating oil dynamic viscosity at high shear [Pas], $\delta_{p}$ - the coefficient determining changes in viscosity as a function of pressure $\left[\mathrm{Pa}^{-1}\right], \delta_{T}$ - the coefficient determining changes in viscosity as a function of temperature $\left[\mathrm{K}^{-1}\right], T_{o}$ - reference temperature $[\mathrm{K}], a, a_{1}$ - the diameters of the shaft (see Fig. 1a), $d, n$ - coefficients of the Cross model $[\mathrm{s}], \dot{\gamma}-$ shear rate $\left[\mathrm{s}^{-1}\right]$.

By applying the condition (5) to the appropriate equations of momentum conservation, we obtain the expected function of the randomly variable velocity vector component in the longitudinal direction:

$$
E X\left[v_{3}(\varphi, y, \zeta)\right]=\left(\frac{1}{h_{3}(\zeta)} \frac{\partial E X(p)}{\partial \zeta}\right) A_{\eta} .
$$

Integration of the continuity equation for the boundary condition (4) i.e. $E X\left(v_{2}\right)=0$ for $y=0$, gives the following form of the expected function of the randomly variable velocity vector component in the gap height direction $y$ :

$$
E X\left[v_{2}(\varphi, y, \zeta)\right]=-\int_{0}^{y} \frac{1}{h_{1}(\zeta)} \frac{\partial E X\left(v_{1}\right)}{\partial \varphi} d y-\int_{0}^{y} \frac{1}{h_{3}(\zeta)} \frac{\partial\left[h_{1} E X\left(v_{3}\right)\right]}{\partial \zeta} d y .
$$

Now we will substitute functions (8), (9) to the solution (10). We apply the boundary condition (4) in the form: $E X\left(v_{2}\right)=0$ for $y=E X\left(\varepsilon_{T}\right)$, i.e. the boundary condition for on the component of the oil velocity vector in the gap height direction $y$. Hence, we get the following stochastic modified Reynolds type equations, determining the expected function $E X[p(\varphi, \zeta)]$ of randomly variable hydrodynamic pressure:

$$
\begin{gathered}
\frac{h_{1}\left(\zeta_{1}\right)}{h_{3}\left(\zeta_{1}\right)} \frac{\partial}{\partial \zeta}\left[\frac{h_{1}\left(\zeta_{1}\right)}{h_{3}\left(\zeta_{1}\right)}\left(\frac{\partial E X(p)}{\partial \zeta}\right)\left(\int_{0}^{E X\left(\varepsilon_{T}\right)} A_{\eta} d y\right)\right]+\frac{\partial}{\partial \varphi}\left[\left(\frac{\partial E X(p)}{\partial \varphi}\right)\left(\int_{0}^{E X\left(\varepsilon_{T}\right)} A_{\eta} d y\right)\right]= \\
=\omega\left(h_{1}\left(\zeta_{1}\right)\right)^{2} \frac{\partial}{\partial \varphi}\left[\int_{0}^{E X\left(\varepsilon_{T}\right)} A_{s} d y-E X\left(\varepsilon_{T}\right)\right] .
\end{gathered}
$$


Substituting the expected functions of the oil velocity vector components (6), (9) into the energy equation, for the constant heat conduction $\kappa$ coefficient, after transformations, we obtain the following differential equation, which allow us to determine the function of the expected temperature:

$$
\frac{\partial^{2} E X(T)}{\partial y^{2}}+\frac{E X\left(\eta_{a}\right)}{\kappa}\left\{\left[\left(\frac{1}{h_{1}\left(\zeta_{1}\right)} \frac{\partial E X(p)}{\partial \varphi}-M_{1}\right) \frac{\partial A_{\eta}}{\partial y}-\omega h_{1}\left(\zeta_{1}\right) \frac{\partial A_{s}}{\partial y}\right]^{2}+\left[\left(\frac{1}{h_{3}\left(\zeta_{1}\right)} \frac{\partial E X(p)}{\partial \zeta}-M_{3}\right) \frac{\partial A_{\eta}}{\partial y}\right]^{2}\right\}=0,
$$

for: $0 \leq \varphi \leq 2 \pi,-b \leq \zeta \leq+b, 0 \leq y \leq E X\left(\varepsilon_{T}\right), E X\left(\varepsilon_{T}\right)=E X\left[\varepsilon_{T}(\varphi, \zeta)\right], E X\left(\eta_{a}\right)=E X\left[\eta_{a}(\varphi, y, \zeta)\right]$,

The determination of the expected function $\operatorname{EX}[T(\varphi, y, \zeta)]$ of randomly variable temperature, from the second order differential equation (12), requires two boundary conditions. The declines and increments of the expected temperature function below and above the characteristic temperature $T_{0}$ finally give a constant temperature value $f_{c}$ at the shaft (moving) surface and unknown value of temperature changes $f_{p}(\varphi, \zeta)$ at the sleeve (not moving) surface. Thus, the two boundary conditions are in the following form:

$$
\begin{array}{ccc}
E X\left[T(\varphi, y, \zeta)=T_{0}+f_{c}\right. & \text { for } & y=0, \\
E X\left[T(\varphi, y, \zeta)=T_{0}+f_{p}(\varphi, \zeta)\right. & \text { for } & y=E X\left(\varepsilon_{T}\right) .
\end{array}
$$

To designate an unknown temperature function $f_{p}(\varphi, \zeta)$ at bearing sleeve surface, we use the condition of the heat flux density $q_{c}$, which is transported from the shaft surface, through the lubricating oil layer, to the bearing sleeve surface. This condition has the following form:

$$
\kappa \frac{\partial E X(T)}{\partial y}=-q_{c} \text { for } y=0 .
$$

\section{Random friction forces, friction coefficient and load carrying capacity}

The components of expected random functions of friction forces in curvilinear $\varphi, \zeta$ directions occurring in parabolic bearing have the following forms:

$$
\begin{aligned}
& E X\left(F_{R 1}\right)=\iint_{\Omega}\left(E X\left(\eta_{T}\right) \frac{\partial E X\left(v_{1}\right)}{\partial y}\right)_{y=E X\left(\varepsilon_{T}\right)} a \cos ^{3}\left(\Lambda_{p 1} \zeta_{1}\right) \sqrt{1+4\left(\Lambda_{p 1} / L_{1}\right)^{2} \sin ^{2}\left(\Lambda_{p 1} \zeta_{1}\right)} d \varphi d \zeta, \\
& E X\left(F_{R 3}\right)=\iint_{\Omega}\left(E X\left(\eta_{T}\right) \frac{\partial E X\left(v_{3}\right)}{\partial y}\right)_{y=E X\left(\varepsilon_{T}\right)} a \cos ^{3}\left(\Lambda_{p 1} \zeta_{1}\right) \sqrt{1+4\left(\Lambda_{p 1} / L_{1}\right)^{2} \sin ^{2}\left(\Lambda_{p 1} \zeta_{1}\right)} d \varphi d \zeta,
\end{aligned}
$$

where: $0 \leq \varphi \leq 2 \pi,-b \leq \zeta \leq+b, 0 \leq y \leq E X\left(\varepsilon_{T}\right), E X\left[\eta_{a}(\varphi, y, \zeta)\right]$ - expected functions of oil viscosity, $E X\left[\varepsilon_{T}(\varphi, \zeta)\right]$ - expected functions of random gap height, $\Omega(\varphi, \zeta)$ - lubrication surface.

The expected value of the bearing load carrying capacity $C[\mathrm{~N}]$, which acts in the opposite direction to the bearing load $\boldsymbol{W}[\mathrm{N}]$, is determined from the following relation:

$$
E X(C)=\sqrt{\left[\int_{-b}^{+b}\left(\int_{0}^{\varphi_{k}} E X[p(\varphi, \zeta)] a \cos ^{2}\left(\Lambda_{p 1} \zeta_{1}\right)(\sin \varphi) d \varphi\right) d \zeta\right]^{2}+\left[\int_{-b}^{+b}\left[\int_{0}^{\varphi_{k}} E X[p(\varphi, \zeta)] a \cos ^{2}\left(\Lambda_{p 1} \zeta_{1}\right)(\cos \varphi) d \varphi\right) d \zeta\right]^{2}},
$$

for $0 \leq \varphi \leq \varphi_{k}<2 \pi,-b \leq \zeta \leq+b$ where: $\varphi_{k}$ is the angular coordinate of the oil film end location, $2 b-$ the length of the sleeve.

The $E X(p)$ is expected value of stochastic hydrodynamic pressure. Using the Coulomb's Law of friction, the dimensionless, randomly variable coefficient of friction, in the parabolic coordinate system, has the following form: 


$$
\mu=\frac{\left|\mathbf{e}_{1} E X\left(F_{R 1}\right)+\mathbf{e}_{3} E X\left(F_{R 3}\right)\right|}{E X(C)},
$$

where $\boldsymbol{e}_{1}, \boldsymbol{e}_{3}$ are the unit vectors in circumferential $\varphi$ and longitudinal $\zeta$ directions.

\section{Numerical calculations}

The numerical calculations of hydrodynamic pressure were made using the finite difference method and subsequent approximations, by solving the equation (11). The calculations were made by simulating the changes in the lubrication gap height due to the surface roughness. The simulated changes in the height of the lubrication gap $\varepsilon_{p}(1+\delta)$ were, as follows: $(1+\delta)=0.80$; $0.85 ; 0.90 ; 0.95 ; 1.00 ; 1.05 ; 1.10 ; 1.15 ; 1.20$, of the nominal value $\varepsilon_{p}$. In the first calculation step, the constant dynamic viscosity of the oil was assumed. After obtaining the hydrodynamic pressure distribution, the values of temperature were determined. In the second calculation step, the 3D dynamic viscosity distribution, calculated by taking into account the influence of pressure, temperature and shear rate, was obtained. Then, the hydrodynamic pressure and temperature distributions were again determined. The calculations were continued in the next calculation steps until the difference in the determined values between successive steps did not differ more than $0.5 \%$. The calculations were performed in the Mathcad 15 program using authors own calculation procedures. Having the final hydrodynamic pressure distributions, the load carrying capacities, friction forces and friction coefficients were calculated, for the relative eccentricities $\lambda=0.4$; $\lambda=0.6 ; \lambda=0.8$, for the bearing with dimensionless length of $L_{1}=1$. The assumed values in the calculations are: the shaft diameters $a=0.035 \mathrm{~m}$ and $a_{1}=0.032 \mathrm{~m}$, the angle between shaft rotation axis and sleeve axis $\gamma=0$, the relative radial clearance $\psi=0.001$, the angular speed of the shaft $\omega=300 \mathrm{~s}^{-1}$, the coefficient describing viscosity changes due to temperature $\delta_{T}=0.04138 \mathrm{~K}^{-1}$ and the coefficient describing changes of viscosity due to pressure $\delta_{p}=-3.706 \cdot 10^{-6} \mathrm{~Pa}^{-1}$. The characteristic dimensional value of dynamic viscosity at the reference temperature $T_{o}=363 \mathrm{~K}$, was $\eta_{o}=0.01358$ Pas, while viscosity for high-pressure $\eta_{h p}=0.026192$ Pas and the viscosity for high shear $\eta_{\infty}=0.01035$ Pas. The coefficients of Cross model were $d=0.0075$ and $n=0.60073$. The value of heat conduction coefficient was of $\kappa=0.15 \mathrm{~W} / \mathrm{mK}$.

The values of the load carrying capacity, calculated with the equation (16), as a function of simulated changes in the height of the lubrication gap, are presented in Fig. 2, while the values of the friction force determined with the equation (15), are shown in Fig. 3. The coefficient of friction (17), as a function of simulated changes in the lubrication gap height, is shown in Fig. 4.

The relative changes of load carrying capacities $\Delta C$, friction forces $\Delta F_{R}$ and coefficients of frictions $\Delta \mu$ for the simulated changes in the lubrication gap height $\varepsilon_{p}\left(1+\delta_{1}\right)$, as a result of taking into account the surface roughness, are shown in Tab. 1. The relative changes of the described values have been calculated according to the following formulas:

$$
\begin{gathered}
\Delta C=\frac{C\left(\varepsilon_{p}\right)-C\left[\varepsilon_{p}\left(1+\delta_{1}\right)\right]}{C\left(\varepsilon_{p}\right)} 100 \%, \Delta F_{R}=\frac{F_{R}\left(\varepsilon_{p}\right)-F_{R}\left[\varepsilon_{p}\left(1+\delta_{1}\right)\right]}{F_{R}\left(\varepsilon_{p}\right)} 100 \%, \\
\Delta \mu=\frac{\mu\left(\varepsilon_{p}\right)-\mu\left[\varepsilon_{p}\left(1+\delta_{1}\right)\right]}{\mu\left(\varepsilon_{p}\right)} 100 \% .
\end{gathered}
$$

\section{Observations and conclusions}

The obtained results of numerical calculations presented in Figs. 2-4 and in Tab. 1, let us deduce that: 
- reduction of the lubrication gap height by $5 \%$ results in an increase of load carrying capacity by $11-12 \%$ on average, while friction forces by $6-7 \%$, but a decrease in the coefficient of friction by $4.4-4.7 \%$,

- reduction of the lubrication gap height by $20 \%$ results in an increase of load carrying capacity by $58-62 \%$ on average, while friction forces by $30-32 \%$, but a decrease in the coefficient of friction by $18-19 \%$,

- increasing the height of the lubrication gap by $5 \%$ causes a drop in the load carrying capacity of $9.6-10.4 \%$, while drop in the friction force value is $5.7-6.1 \%$, and causes an increase in the coefficient of friction by $4.3-4.7 \%$,

- increasing the height of the lubrication gap by $20 \%$ causes a drop in the load carrying capacity of $31-34 \%$ and drop in the friction force value of $19.8-21.4 \%$, and causes an increase in the coefficient of friction by $17-19 \%$.

a)

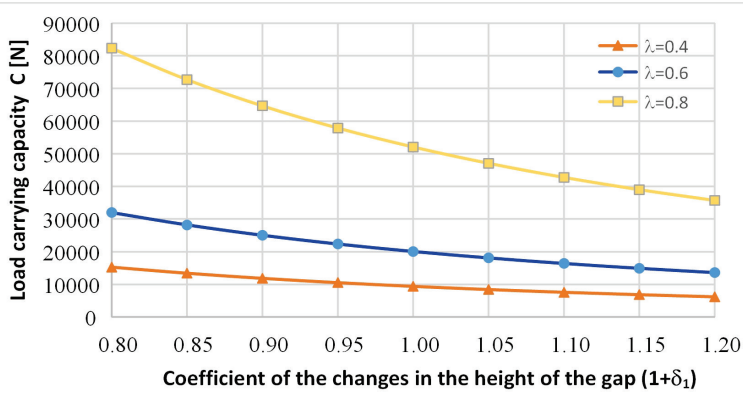

b)

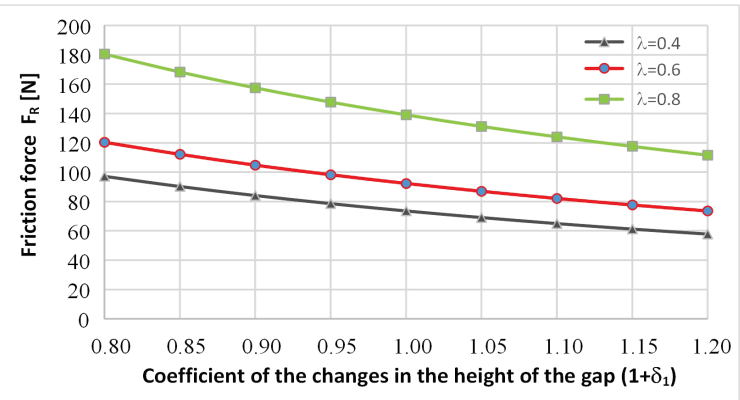

c)

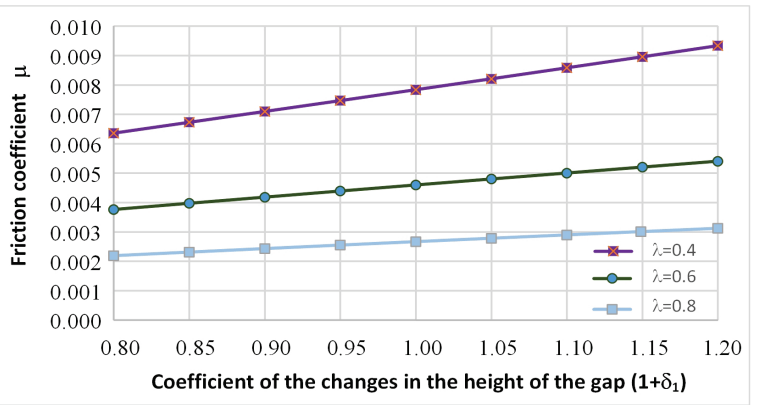

Fig. 2. Load carrying capacity (a), friction force (b), friction coefficient (c) as a function of simulated changes of lubrication gap height, for the relative eccentricities: $\lambda=0.4, \lambda=0.6, \lambda=0.8$

Tab. 1. The relative changes in the values of load carrying capacity $\Delta C$, friction force $\Delta F_{R}$ and friction coefficient $\Delta \mu$ for the simulated changes of lubrication gap height $\varepsilon_{p}\left(1+\delta_{1}\right)$

\begin{tabular}{|c|c|c|c|c|c|c|c|c|c|c|}
\hline \multirow{2}{*}{$\begin{array}{c}\text { Change } \\
\text { of gap } \\
\text { height }\end{array}$} & \multicolumn{3}{|c|}{ Relative eccentricity } & \multicolumn{3}{c|}{ Relative eccentricity } & \multicolumn{3}{c|}{ Relative eccentricity } \\
\cline { 2 - 10 } & $\lambda=0.4$ & \multicolumn{2}{|c|}{$\begin{array}{c}\lambda=0.6 \\
\text { Relative changes of load } \\
\text { carrying capacity } \Delta C[\%]\end{array}$} & \multicolumn{2}{c|}{$\begin{array}{c}\text { Relative changes of friction } \\
\text { force } \Delta F_{R}[\%]\end{array}$} & \multicolumn{2}{c|}{$\begin{array}{c}\text { Relative changes of friction } \\
\text { coefficient } \Delta \mu[\%]\end{array}$} \\
\hline $0.80 \cdot \varepsilon_{p}$ & -62.74 & -59.39 & -58.05 & -32.06 & -30.39 & -29.76 & 18.86 & 18.15 & 17.90 \\
\hline $0.85 \cdot \varepsilon_{p}$ & -42.92 & -40.56 & -39.61 & -22.66 & -21.51 & -20.99 & 14.14 & 13.54 & 13.33 \\
\hline $0.90 \cdot \varepsilon_{p}$ & -26.19 & -24.76 & -24.17 & -14.29 & -13.49 & -13.23 & 9.44 & 9.00 & 8.84 \\
\hline $0.95 \cdot \varepsilon_{p}$ & -12.12 & -11.41 & -11.14 & -6.77 & -6.41 & -6.25 & 4.73 & 4.48 & 4.38 \\
\hline $1.00 \cdot \varepsilon_{p}$ & - & - & - & - & - & - & - & - & - \\
\hline $1.05 \cdot \varepsilon_{p}$ & 10.39 & 9.82 & 9.58 & 6.13 & 5.80 & 5.68 & -4.74 & -4.43 & -4.34 \\
\hline $1.10 \cdot \varepsilon_{p}$ & 19.38 & 18.29 & 17.88 & 11.72 & 11.09 & 10.78 & -9.51 & -8.85 & -8.61 \\
\hline $1.15 \cdot \varepsilon_{p}$ & 27.21 & 25.71 & 25.10 & 16.80 & 15.91 & 15.46 & -14.30 & -13.22 & -12.84 \\
\hline $1.20 \cdot \varepsilon_{p}$ & 34.06 & 32.24 & 31.43 & 21.45 & 20.33 & 19.77 & -19.12 & -17.59 & -17.03 \\
\hline
\end{tabular}


The influence of the relative eccentricity on the relative changes of bearing load carrying capacity, friction force and coefficient of friction, is small, i.e. on the order of a few percent.

The stochastic changes in the lubrication gap height, due to surface roughness or other factors, have a significant impact on the operational parameters of the hydrodynamic slide bearings; therefore, it should be taken into account during simulations and design of slide bearings.

\section{References}

[1] Bartz, W. J., u.a., Gleitlagertechnik, Expert Verlag, Grafenau 1981.

[2] Dai, R. X., Dong, Q., Szeri, A. Z., Approximations in hydrodynamic lubrication, Transactions of the ASME, Journal of Tribology, Vol. 114, pp. 14-25, 1992.

[3] Dunn, J. E., Rajagopal, K. R., Fluids of differential type: critical review and thermodynamic analysis, International Journal of Engineering Science, Vol. 33 (5), pp. 689-729, 1995.

[4] Fisz, M., Rachunek prawdopodobieństwa i statystyka matematyczna, PWN, Warszawa 1967.

[5] Galindo-Rosales, F. J, Rubio-Hernández, F. J., Sevilla, A., An apparent viscosity function for shear thickening fluids, Journal of Non-Newtonian Fluid Mechanics, Vol. 166, pp. 321-325, 2011.

[6] Guha, S. K., Analysis of steady-state characteristics of misaligned hydrodynamic journal bearings with isotropic roughness effect, Elsevier, Tribology International, Vol. 33, pp. 1-12, 2000.

[7] Hashimoto, H., Surface Roughness effects in high-speed hydrodynamic journal bearings, Transactions of the ASME, Journal of Tribology, Vol. 119, pp. 776-740, 1997.

[8] Helwig, Z., Elementy rachunku prawdopodobieństwa i statystyki matematycznej, PWN, Warszawa 1977.

[9] Miszczak, A., Analiza hydrodynamicznego smarowania łożysk ślizgowych cieczami o właściwościach nienewtonowskich, Monografia, Wydawnictwo Naukowe Instytutu Technologii Eksploatacji-PIB w Radomiu, pp. 1-336, Radom 2019.

[10] Wierzcholski, K., Miszczak, A., Electro-magneto-hydrodynamic lubrication, Open Physics, Vol. 16 (1), pp. 285-291, 2018. 